\title{
Reply to commentary to: factors influencing endoscopic dacryocystorhinostomy outcome
}

\author{
Kazuhiro Nomura ${ }^{1,2}$ (D) Kazuya Arakawa $^{1,3} \cdot$ Mitsuru Sugawara $^{3} \cdot$ Hiroshi Hidaka $^{1} \cdot$ \\ Jun Suzuki ${ }^{1,3} \cdot$ Yukio Katori $^{1}$
}

Received: 27 July 2017 / Accepted: 8 August 2017 / Published online: 21 August 2017

(c) Springer-Verlag GmbH Germany 2017

\section{Dear Editor,}

We thank the reviewers for their comments on our article entitled "Factors influencing endoscopic dacryocystorhinostomy outcome" [1]. There are other factors apart from the four factors listed in the article (age, obstruction site, stent insertion and duration of symptom). Based on the number of cases and failure rate, only four predictive variables could have been fitted reliably for logistic regression analysis in the study [2]. We thought that the four factors mentioned above were more likely to be associated with the outcome than other factors including operator and anatomical variations of the nasal cavity.

In our series, simultaneous endoscopic sinus surgery was performed in only four cases accompanying sinusitis, and septoplasty was performed in 21 cases to enable access to the surgical site. Performing septoplasty was not associated with poor outcome (odds ratio $0.51,95 \%$ confidence interval $0.11-2.28, p=0.377$ ).

We followed patients for at least 6 months after surgery except in rare cases where the wound healing was apparently good and further follow-up seemed unnecessary. Two

This reply refers to the article available at doi:10.1007/s00405017-4685-6.

Kazuhiro Nomura

kazuhiroe@gmail.com

1 Department of Otolaryngology-Head and Neck Surgery, Tohoku University Graduate School of Medicine, Sendai, Japan

2 Department of Otolaryngology, Tohoku Rosai Hospital, Sendai, Japan

3 Department of Otolaryngology, Tohoku Kosai Hospital, Sendai, Japan surgeons performed the surgeries and the outcomes were not significantly different (odds ratio $0.78,95 \%$ confidence interval $0.25-2.39, p=0.665)$. Subjective symptoms were completely cured in $85.0 \%$ (181/213) of sides and improved in $93.9 \%(200 / 213)$ of sides. Objectively, DCR closed in $4.2 \%(9 / 213)$ of sides.

We agree that there are a myriad of factors contributing to poor outcome. Due to limited patient numbers, only four factors were analyzed.

\section{Compliance with ethical standards}

Conflict of interest The authors declare that they have no conflict of interest.

Ethical approval All procedures performed in studies involving human participants were in accordance with the ethical standards of the institutional research committee and with the 1964 Helsinki declaration and its later amendments. This study is a retrospective chart review.

\section{References}

1. Nomura K, Arakawa K, Sugawara M, Hidaka H, Suzuki J, Katori Y (2017) Factors influencing endoscopic dacryocystorhinostomy outcome. Eur Arch Otorhinolaryngol 274:2773-2777

2. Peduzzi P, Concato J, Kemper E, Holford TR, Feinstein AR (1996) A simulation study of the number of events per variable in logistic regression analysis. J Clin Epidemiol 49:1373-1379 